\title{
Psychological and Psychophysiological Effects of Recuperative Music Postexercise
}

\author{
COSTAS I. KARAGEORGHIS ${ }^{1}$, ANDREW C. BRUCE ${ }^{1}$, SUZANNE T. POTTRATZ ${ }^{2}$, REBECCA C. STEVENS ${ }^{1}$, \\ MARCELO BIGLIASSI ${ }^{1}$, and MARK HAMER ${ }^{3}$ \\ ${ }^{1}$ Department of Life Sciences, Brunel University London, Uxbridge, UNITED KINGDOM; ${ }^{2}$ Department of Psychology, \\ East Central University, Ada, OK; and ${ }^{3}$ School of Sport, Exercise and Health Sciences, Loughborough University, \\ Loughborough, UNITED KINGDOM
}

\begin{abstract}
KARAGEORGHIS, C. I., A. C. BRUCE, S. T. POTTRATZ, R. C. STEVENS, M. BIGLIASSI, and M. HAMER. Psychological and Psychophysiological Effects of Recuperative Music Postexercise. Med. Sci. Sports Exerc., Vol. 50, No. 4, pp. 739-746, 2018. Purpose: Few studies have examined the psychological and psychophysiological effects of recuperative music after exhaustive exercise. The main purpose of the present study was to examine the effects of two music conditions compared with a no-music control on psychological and psychophysiological recovery processes after exercise. Methods: A randomized, fully counterbalanced, crossover design was used. Core affect, salivary cortisol, heart rate, and blood pressure were measured before exhaustive exercise, immediately after, and in 10-, 20-, and 30-min intervals during passive recovery ( 21 women and $21 \mathrm{men}$; $20.9 \pm 1.7 \mathrm{yr}$ ) over three separate trials (slow, sedative music; fast, stimulative music; no-music control). The exercise task entailed incremental cycle ergometry performed at $75 \mathrm{rpm}$ with an increase in intensity of $22.5 \mathrm{~W} \cdot \mathrm{min}^{-1}$ at the end of each minute until exhaustion. Data were analyzed using mixed-model 3 (condition) $\times 4$ (time) $\times$ 2 (gender) MANOVA/ANCOVA. Results: The largest decline in affective arousal between active and passive recovery phases was evident in the slow, sedative condition $\left(\eta_{\mathrm{p}}{ }^{2}=0.50\right)$. Women had a more pronounced reduction in arousal than did men in the slow, sedative music condition. Heart rate measures showed that fast, stimulative music inhibited the return of heart rate toward resting levels $\left(\eta_{\mathrm{p}}{ }^{2}=0.06\right)$. Similarly, salivary cortisol levels tended to be lower in response to slow, sedative music $\left(\eta_{\mathrm{p}}{ }^{2}=0.11\right)$. There was a main effect of condition for affective valence indicating that the slow, sedative condition elicited more positive affective responses compared with the control and fast, stimulative conditions $\left(\eta_{\mathrm{p}}{ }^{2}=0.12\right)$. Conclusions: The present findings support the notion that slow, sedative music can expedite the recovery process immediately after strenuous exercise. Key Words: AFFECT, CORTISOL, ENTRAINMENT, RECOVERY, PSYCHOBIOLOGY, SEDATION
\end{abstract}

I t has been claimed that "music hath charms to soothe the savage beast" (c.f. Orpheus) but a number of contemporary researchers have been captivated by the notion of whether it might also "hath charms" to soothe recovery from strenuous physical exercise $(1,2)$. Recovery can be defined as the return to baseline or resting state and its enhancement has implications for the degree to which recreationally active individuals enjoy and adhere to exercise (3). Most of the literature addressing the psychological and psychophysiological

Address for correspondence: Costas I. Karageorghis, Ph.D., Department of Life Sciences, Brunel University London, Uxbridge, UB8 3PH, United Kingdom; E-mail: costas.karageorghis@brunel.ac.uk.

Submitted for publication July 2017.

Accepted for publication November 2017.

0195-9131/18/5004-0739/0

MEDICINE \& SCIENCE IN SPORTS \& EXERCISE ${ }_{\circledast}$

Copyright $(2017$ The Author(s). Published by Wolters Kluwer Health, Inc. on behalf of the American College of Sports Medicine. This is an open access article distributed under the Creative Commons Attribution License 4.0 (CCBY), which permits unrestricted use, distribution, and reproduction in any medium, provided the original work is properly cited.

DOI: 10.1249/MSS.0000000000001497

effects of music in the exercise domain has focused on pretask and in-task applications $(4,5)$. To date, scant attention has been given to investigation of the posttask application of music (6). In an exercise context, posttask music is proposed to serve a recuperative role and aid recovery from stressful tasks, such as maximal exercise protocols (7). One important distinction in this literature, not always made explicit by researchers, has entailed the use of music for movement-based recovery, known as active recovery, and static-based recovery, known as passive recovery (8). The protocol of the present study entailed both active and passive recovery, with the primary focus placed on a 30-min passive recovery phase.

Passive recovery. In the inaugural study into the effects of posttask music in the exercise domain, Jing and Xudong (9) applied a sedative instrumental piece to aid the passive recovery of male students for 15 min after an exhaustive cycle ergometer trial. Decreases in heart rate (HR), urinary protein (indicative of postexercise kidney function), and RPE were greater in the music group when compared with a control group that recovered in a supine position for 15 min without music. Subsequently, Savitha et al. (10) showed that slow music accelerated the hemodynamic (blood pressure and HR) recovery after $5 \mathrm{~min}$ of intense treadmill running in comparison to both fast music ( $17 \%$ difference) 
and a control (32\% difference). Interestingly, there were no differences between the genders in terms of responsiveness to the two music conditions.

Underlying concepts and mechanisms. Over the past two decades, there has been a flurry of scientific activity in the quest to shed light on the mechanisms that underlie the effects of music in physical activity and other contexts $(4,5,11,12)$. One of the most oft-cited uses of music by exercisers entails the regulation or modulation of affective valence and arousal, as well as the inducement of specific emotions (e.g., happiness, liveliness, calmness, or aggression [13]). In terms of the arousal dimension of core affect (i.e., "the most elementary consciously accessible affective feelings"; [14, p. 806]), there is an association between the stimulative or sedative qualities of a piece of music (especially tempo and volume [15]) and the function that it serves in different listening situations $(16,17)$.

Exercising at a severe intensity causes a sharp decline in affective valence (i.e., how pleasant one feels) that is coupled with high levels of psychomotor arousal (18). For recovery, therefore, it would seem intuitive that slow, sedative music would be most efficacious when the essential goal is to return the body to homeostasis. However, it is notable from the exercise-related affect literature that affective valence returns to baseline levels almost immediately after cessation of severe exercise - the so-called affective rebound - whereas affective arousal takes considerably longer to return to baseline levels $(1,19)$. Accordingly, the role of slow, sedative music in the postexercise phase may be more salient in terms of down-regulating affective arousal than up-regulating affective valence.

In Juslin's (12) unified theory of emotional responses to music, the brain stem reflex is a proposed mechanism in the regulation of arousal. This entails the process by which the fundamental acoustic properties of music stimulate responses by signaling a potentially important or urgent event. For example, fast, loud music would automatically stimulate the listener by activating the central nervous system irrespective of how the music is subsequently appraised. This stimulation results in elevated HR, blood pressure, body temperature, skin conductance, and muscle tension (20). Soft, slow music has the converse effect and thus decreases levels of sympathetic arousal. When there is a desire to expedite recovery after an intense bout of exercise or even in between bouts (e.g., during interval training), the potential of a musical stimulus to down-regulate psychomotor arousal is particularly salient (7). In mechanistic terms, this is closely allied to the biomusicological process of rhythmic entrainment, wherein bodily pulses such as heart/respiration rate and brainwaves are drawn into a common oscillation with musical tempo $(12,17)$.

Purpose and hypotheses. The main purpose of the present study was to examine the effects of two music conditions and a no-music control on psychological and psychophysiological recovery processes after exhaustive cycle ergometer exercise. A secondary purpose was to explore the moderating influence of gender in participants' responsiveness to the musical interventions, given recent indications that the effects are gender invariant (10). It was hypothesized that a slow, sedative condition would be most beneficial in terms of a range of psychological and psychophysiological recoveryrelated indices when compared with both a fast, stimulative condition and a no-music control. For gender as a potential moderator, the null hypothesis was tested given the lack of moderation evidenced in past research (e.g., 10).

\section{METHODS}

\section{Participants}

An a priori power analysis using $\mathrm{G}^{*}$ Power 3.1 (21) indicated that 40 participants would suffice on the basis of a small effect size for HR in a similar study $\left(\eta_{\mathrm{p}}{ }^{2}=0.04\right.$ [9] $)$ at an alpha level of 0.05 and a desired power of 0.90 for a crossover design. To achieve counterbalancing through the six different routes of the protocol, a minimum of 42 participants were required. With institutional ethics committee approval and written informed consent, 42 recreationally active students from Brunel University London (21 women and $21 \mathrm{men} ; 20.9 \pm 1.7 \mathrm{yr}$ ) were recruited. Participants were Caucasian, brought up in the United Kingdom, and homogeneous in terms of age and sociocultural background (22).

\section{Equipment and Materials}

General Health Questionnaire. The General Health Questionnaire (GHQ-28 [23]) was used to assess trait anxiety and chronic psychological stress. It comprises 28 questions divided into four, seven-item sections: (A) somatic symptoms, (B) anxiety and insomnia, (C) social dysfunction, and (D) severe depression. The respondent answers the questions in regard to their general health over the "past few weeks." The reliability and validity of the instrument have been tested extensively across various populations (24).

Affect Grid. The Affect Grid (25) was used to measure core affect (i.e., dimensions of pleasure and arousal). This instrument comprises a $9 \times 9$ grid, with the horizontal dimension representing affective valence and the vertical dimension representing affective arousal. Participants place a single " $\mathrm{X}$ " in one of the 81 cells, and this response is scored along both the two dimensions. The Affect Grid has shown high reliability $(0.98$ for pleasure scores, 0.97 for arousal scores) and adequate convergent, discriminant, and predictive validity when compared with Mehrabian and Russell's (26) scales of pleasure/arousal. It has also demonstrated acceptable concurrent validity with closely related instruments such as the Feeling Scale and Felt Arousal Scale (27).

\section{Apparatus}

A stadiometer was used to measure height (Seca 217), and body mass was measured using weighing scales (Model 798 Electronic Scales). Laboratory conditions were standardized 
by maintaining constant temperature and relative humidity. Barometric pressure ( $\mathrm{mm} \mathrm{Hg}$ ) was measured using a mercury column barometer (Philip Harris Ltd, Mercury Column Barometer). Each test was performed on a cycle ergometer (Ergomedic 874E Cycle Ergometer). Music was delivered via an Apple iPod (iPod 30GB, G5) with standard in-ear Apple headphones. The volume was standardized at level 10 , which is deemed safe from an audiological perspective (28). Saliva samples were collected using salivettes (Sarsted) that were stored at $-80^{\circ} \mathrm{C}$ until analysis. Levels of cortisol were assessed using a time-resolved immunoassay with fluorescence detection at the University of Dresden, Germany. The intra-assay and interassay coefficients of variation were less than $8 \%$. Each participant's HR was taken using a chest transmitter attached to the sternum and a sensor (Polar Accurex Plus Heart Rate Monitor). Blood pressure was taken by means of an upper-arm blood pressure monitor (Accoson Freestyle Mercury Sphygmomanometer).

\section{Procedure}

Participants came to the laboratory $1 \mathrm{wk}$ before the start of testing, for a habituation session. They were fully familiarized with the testing protocol and screened for psychological distress using the GHQ-28. They were informed that they were not permitted to eat for up to $2 \mathrm{~h}$ before the upcoming trials and were asked to refrain from high-intensity exercise for up to $1 \mathrm{~d}$ before each trial. They were also instructed not to drink caffeine-based drinks, brush their teeth, or smoke for $2 \mathrm{~h}$ before each trial, because such activities can invalidate measures of salivary cortisol. Each test took place around the same time of day and in the same laboratory with at least $2 \mathrm{~d}$ of rest between trials.

A within-subjects design was used, wherein each participant was administered a control condition and two experimental conditions in a fully counterbalanced order. Upon entering the laboratory, the participant was asked to sit in silence for $10 \mathrm{~min}$ after which her/his core affect, salivary cortisol, resting HR, and blood pressure were measured. $\mathrm{S} / \mathrm{he}$ then completed a 3-min warm-up on the cycle ergometer at a pedal rate of $50 \mathrm{rpm}$. After the warm-up, the participant was required to cycle at a constant speed of $75 \mathrm{rpm}$ and a 300 -g disk was immediately added to the ergometer weight basket. At the end of each minute, a further 300 -g disk was added to the weight basket, thus providing the equivalent of an increase in resistance of $22.5 \mathrm{~W} \cdot \mathrm{min}^{-1}$. When the participant could no longer maintain a speed of $70 \mathrm{rpm}$ for a period greater than $5 \mathrm{~s}$ ( $5 \mathrm{rpm}$ lower than the desired speed), it was determined that $\mathrm{s} / \mathrm{he}$ had reached exhaustion and the test was terminated.

The participant was instructed to continue cycling for $3 \mathrm{~min}$ at $50 \mathrm{rpm}$ with no weights in the basket, as a form of active recovery. Concurrently, the participant was provided with headphones for one of the three conditions (fast, stimulative music, slow, sedative music, or no-music control). The total recovery period of $33 \mathrm{~min}$ comprised a 3-min active recovery followed by a $30-\mathrm{min}$ period of passive recovery, for which the participant was instructed to move to a nearby comfortable chair, with the in-ear phones still in place. The slow, sedative music condition met published criteria for recuperative music (29), whereas the fast, stimulative music condition was representative of music typically used during exercise $(13,30)$. The fast, stimulative music condition comprised nine tracks with an average tempo of $129 \mathrm{bpm}$. The slow, sedative music condition comprised two longer tracks that had an average tempo of $71 \mathrm{bpm}$ and were thus close to resting HR for a healthy adult. The participant was also administered the Affect Grid and had cortisol, HR, and blood pressure measured during the 3-min period of active recovery that immediately followed voluntary exhaustion. Affect Grid, cortisol, HR, and blood pressure measures were retaken at 10-, 20-, and 30-min intervals during passive recovery.

\section{Statistical Analysis}

SPSS 18.02 for Windows was used to analyze the data, and alpha was set at 0.05 to establish statistical significance. Data were examined for univariate outliers, and the relevant tests for parametric assumptions were conducted. The assumptions underlying the use of covariates were also tested [31; pp. 203-205]. Mixed-model 3 (condition) $\times 5$ (time) $\times$ 2 (gender) ANCOVA was used to analyze cortisol, HR, systolic blood pressure (sysBP), and diastolic blood pressure (diaBP). To account for the potential confound of baseline variability, the mean of the pretest measure of each variable was used as a covariate. Nonetheless, to maximize differences across conditions for affective valence and arousal, the two covariates were not used and a mixed-model 3 (condition) $\times 5$ (time) $\times 2$ (gender) MANOVA was computed to analyze the composite of these two variables. After examination of omnibus statistics, Bonferroni-adjusted pairwise comparisons were used to identify where differences lay.

\section{RESULTS}

\section{Initial Data Screening and Diagnostic Tests}

Initial data screening revealed 10 univariate outliers ( 8 for cortisol and 2 for HR) that were altered by assigning the outlying case a raw score on the offending variable that was one unit larger or smaller than the next most extreme score in the distribution [31, p. 77]. One case was excluded from the cortisol analysis because of an insufficient volume of saliva in 6 of the 15 salivettes. After examination of standard skewness and kurtosis scores, a square root transformation was applied to the salivary cortisol scores, because the distribution was positively skewed and kurtotic $(P<0.01)$. Moreover, Mauchly's test indicated violations of the sphericity assumption $(P<0.05)$ for within-subject effects of time on HR; of time and condition-time on cortisol and sysBP; of condition, time, and condition-time on diaBP and arousal; and of time and condition-time on affective valence. Therefore, Greenhouse-Geisser adjustments were applied to the relevant $F$ tests. The overall scores of the GHQ-28 indicated that none 
TABLE 1. Descriptive statistics, mixed-model MANOVA for affective valence and arousal, and repeated-measures ANCOVA for salivary cortisol, HR, sysBP, and diaBP.

\begin{tabular}{|c|c|c|c|c|c|}
\hline Dependent Variables & Conditions & Mean & SD & $F_{d f}$ & $\eta_{\mathrm{p}}^{2}$ \\
\hline \multirow[t]{3}{*}{ Affective valence } & Control & 6.11 & 1.02 & & \\
\hline & Slow, sedative music & 6.70 & 0.86 & $5.44^{\star}{ }_{2,80}$ & 0.12 \\
\hline & Fast, stimulative music & 6.22 & 1.25 & & \\
\hline \multirow[t]{3}{*}{ Affective arousal } & Control & 4.54 & 1.01 & & \\
\hline & Slow, sedative music & 3.58 & 0.87 & $40.62^{\star \star} 1.45,58.27$ & 0.50 \\
\hline & Fast, stimulative music & 5.48 & 1.28 & & \\
\hline \multirow[t]{3}{*}{ Cortisol, $\mu \mathrm{g} \cdot \mathrm{dL}^{-1}$} & Control & 10.49 & 6.33 & & \\
\hline & Slow, sedative music & 10.29 & 6.52 & $0.20_{2,76}$ & 0.01 \\
\hline & Fast, stimulative music & 12.15 & 8.23 & & \\
\hline \multirow[t]{3}{*}{ HR, bpm } & Control & 110.52 & 9.42 & & \\
\hline & Slow, sedative music & 109.59 & 9.30 & $0.92_{2,78}$ & 0.02 \\
\hline & Fast, stimulative music & 115.67 & 8.88 & & \\
\hline \multirow[t]{3}{*}{ sysBP, mm Hg } & Control & 122.17 & 7.21 & & \\
\hline & Slow, sedative music & 118.53 & 8.00 & $2.84_{2,78}$ & 0.07 \\
\hline & Fast, stimulative music & 123.35 & 5.52 & & \\
\hline \multirow[t]{3}{*}{ diaBP, $\mathrm{mm} \mathrm{Hg}$} & Control & 73.32 & 9.00 & & \\
\hline & Slow, sedative music & 70.94 & 8.68 & $1.38_{1.53,59.87}$ & 0.03 \\
\hline & Fast, stimulative music & 73.24 & 8.85 & & \\
\hline \multicolumn{6}{|l|}{ Time-gender interaction } \\
\hline Cortisol & $F_{1.28,48.63}=5.41, P=0.017, \eta_{\mathrm{p}}^{2}=0.13$ & & & & \\
\hline \multirow{2}{*}{\multicolumn{6}{|c|}{ Condition-gender interaction }} \\
\hline & & & & & \\
\hline \multirow{2}{*}{\multicolumn{6}{|c|}{ Condition-time interaction }} \\
\hline & & & & & \\
\hline Cortisol & $F_{2.93,111.34}=4.81, P=0.004, \eta_{\mathrm{p}}^{2}=0.11$ & & & & \\
\hline $\begin{array}{l}\text { Affective arousal } \\
\text { HR }\end{array}$ & $\begin{array}{l}F_{4.06,162.41}=11.40, P<0.001, \eta_{\mathrm{p}}{ }^{2}=0.22 \\
F_{0}=2.50, P=0.023, \eta^{2}=0.06\end{array}$ & & & & \\
\hline \multicolumn{6}{|c|}{ Affective variables omnibus statistics } \\
\hline Condition-time & Pillai's trace $=0.24, F_{12,480}=5.36, P<0.001, \eta_{p}^{2}=0.12$ & & & & \\
\hline Condition & Pillai's trace $=0.56, F_{4,160}=15.65, P<0.001, \eta_{\mathrm{p}}^{2}=0.28$ & & & & \\
\hline Time & Pillai's trace $=0.70, F_{6,240}=21.53, P<0.001, \eta_{p}^{2}=0.35$ & & & & \\
\hline Gender & Hotteling's $T=0.01, F_{2,39}=0.22, P=0.799, \eta_{p}^{2}=0.01$ & & & & \\
\hline
\end{tabular}

The $F$ tests for affective arousal, cortisol, sysBP, and sysBP were Greenhouse-Geisser adjusted.

${ }^{*} P<0.05$.

${ }^{* *} P<0.001$.

of the participants were experiencing any major detriments to their general mental health.

\section{Interaction Effects}

In the interests of brevity, only significant $(P<0.05)$ inferential statistics for interaction effects are included in Table 1.

Affective valence. There was no significant higherorder interaction effect of condition-time-gender for affective valence $\left(P=0.684, \eta_{\mathrm{p}}^{2}=0.01\right)$. Similarly, there were no significant two-way interactions of condition-time $(P=$ $\left.0.804, \eta_{\mathrm{p}}{ }^{2}=0.01\right)$, condition-gender $\left(P=0.404, \eta_{\mathrm{p}}{ }^{2}=\right.$ $0.02)$, or time-gender $\left(P=0.590, \eta_{\mathrm{p}}^{2}=0.01\right)$.

Affective arousal. There was no significant higherorder interaction effect of condition-time-gender $(P=$ $\left.0.424, \eta_{\mathrm{p}}{ }^{2}=0.02\right)$ and no two-way interaction of timegender $\left(P=0.361, \eta_{\mathrm{p}}{ }^{2}=0.02\right)$ for affective arousal. There was, however, a significant two-way interaction of conditiontime $\left(P<0.001, \eta_{\mathrm{p}}{ }^{2}=0.22\right)$ and condition-gender $(P=$ $0.040, \eta_{\mathrm{p}}{ }^{2}=0.09$; see Table 1). Examination of standard errors (SEs) indicated that arousal decreased over time in all three conditions, with the largest decline between active and passive recovery evident in the slow, sedative music condition (see Fig. 1). When control was compared with the slow sedative condition, SEs indicated that women had a more pronounced reduction in arousal scores than did men. It is notable that women also reported higher levels of arousal than men in the control condition $\left(M_{\text {diff }}=0.81\right)$.
Salivary cortisol. There was no significant higherorder interaction of condition-time-gender for salivary cortisol $\left(P=0.786, \eta_{\mathrm{p}}{ }^{2}=0.01\right)$ or a two-way interaction of condition-gender $\left(P=0.748, \eta_{\mathrm{p}}{ }^{2}=0.01\right)$. There was, however, a significant interaction effect for condition-time $(P=0.004$, $\eta_{\mathrm{p}}{ }^{2}=0.11$; see Table 1). Examination of SEs indicated that cortisol levels were lower in the slow, sedative music condition compared with the fast, stimulative music condition at time 1 (active recovery). Cortisol levels were also lower in the slow, sedative condition when compared with the fast, stimulative condition at time 3 ( $+20 \mathrm{~min}$; see Fig. 2). There was a significant interaction of time-gender $\left(P=0.017, \eta_{\mathrm{p}}{ }^{2}=\right.$ 0.12; see Fig. 3 and Table 1). Examination of SEs indicated that women's cortisol levels remained relatively stable over

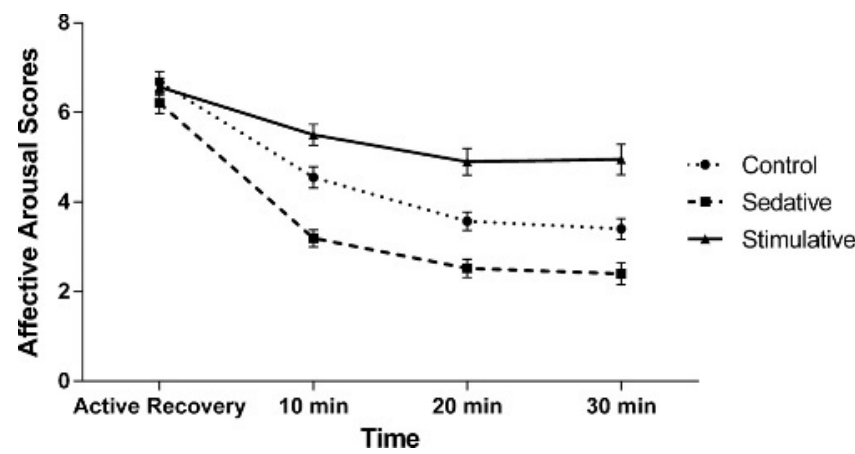

FIGURE 1-Significant condition-time interaction $(P<0.001)$ for af fective arousal. Error bars represent SE. 


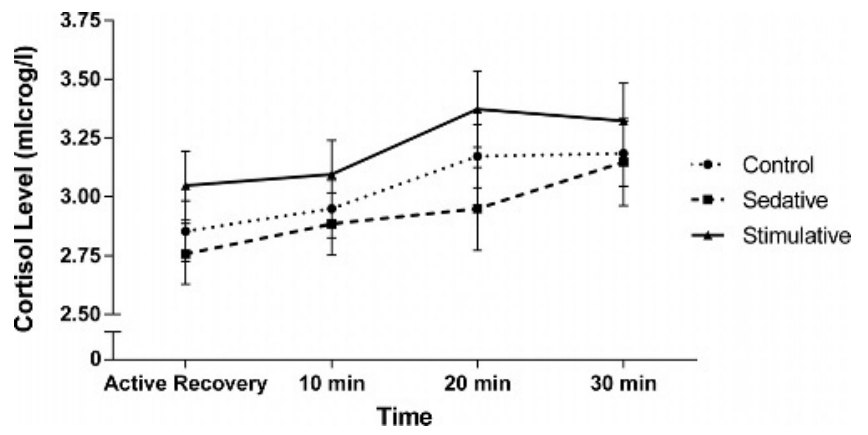

FIGURE 2-Significant condition-time interaction $(P=0.040)$ for salivary cortisol (square root-transformed data). Error bars represent SE.

time, whereas men's increased at time 3 and time $4(+20 \mathrm{~min}$ and +30 min, respectively).

HR. There was no significant higher-order interaction effect of condition-time-gender for HR $\left(P=0.071, \eta_{\mathrm{p}}{ }^{2}=\right.$ $0.05)$. There were also no significant two-way interactions of condition-gender $\left(P=0.185, \eta_{\mathrm{p}}{ }^{2}=0.04\right)$ or time-gender $\left(P=0.250, \eta_{\mathrm{p}}^{2}=0.03\right)$. There was, however, a significant two-way interaction effect of condition-time $\left(P=0.023, \eta_{\mathrm{p}}{ }^{2}=\right.$ 0.06 ; see Table 1). The largest decline between active and passive recovery was evident in the control and slow, sedative music conditions when compared with the fast, stimulative condition (see Fig. 4). HR was more elevated in the fast, stimulative music condition at time 2 , time 3 , and time $4(+10 \mathrm{~min}$, $+20 \mathrm{~min}$, and $+30 \mathrm{~min}$, respectively) when compared with the slow, sedative music and control conditions.

sysBP. There was no significant higher-order interaction effect of condition-time-gender for sysBP $\left(P=0.086, \eta_{\mathrm{p}}{ }^{2}=\right.$ $0.04)$. Similarly, there were no significant two-way interactions for condition-time $\left(P=0.267, \eta_{\mathrm{p}}{ }^{2}=0.03\right)$ or condition-gender $\left(P=0.544, \eta_{\mathrm{p}}{ }^{2}=0.01\right)$. There was, however, a significant twoway interaction for time-gender $\left(P=0.014, \eta_{\mathrm{p}}{ }^{2}=0.12\right.$; see Table 1). Examination of SEs for the interaction indicated that men's sysBP was higher at time 1 (active recovery) and lower at time 3 and time 4 ( $+20 \mathrm{~min}$ and $+30 \mathrm{~min}$, respectively) when compared with women's.

diaBP. There was no significant higher-order interaction of condition-time-gender for diaBP $\left(P=0.941, \eta_{\mathrm{p}}{ }^{2}=0.01\right)$. There were also no two-way interactions for condition-time $\left(P=0.097, \eta_{\mathrm{p}}{ }^{2}=0.05\right)$, condition-gender $\left(P=0.473, \eta_{\mathrm{p}}{ }^{2}=\right.$ $0.02)$, or time-gender $\left(P=0.680, \eta_{\mathrm{p}}^{2}=0.01\right)$.

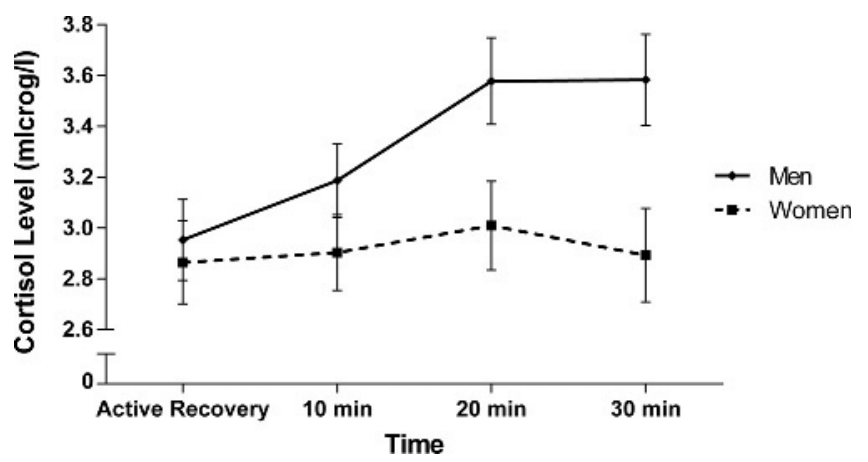

FIGURE 3-Significant time-gender interaction $(P=0.017)$ for salivary cortisol (square root-transformed data). Error bars represent SE.

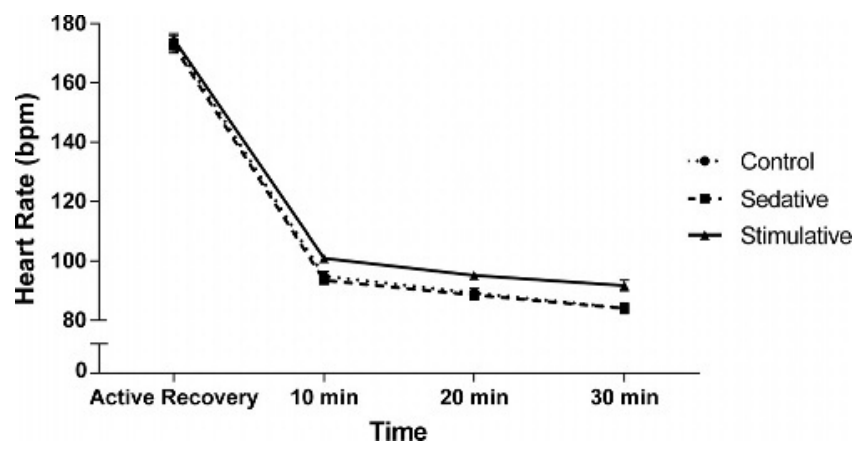

FIGURE 4-Significant condition-time interaction $(P=\mathbf{0 . 0 2 3})$ for HR. Error bars represent SE.

\section{Main Effects}

Inferential statistics for all main effects are presented in Table 1 along with descriptive statistics.

Affective valence. There was a main effect of condition for affective valence $\left(P=0.006, \eta_{\mathrm{p}}{ }^{2}=0.12\right.$; see Table 1$)$, with pairwise comparisons indicating that slow, sedative music elicited more positive affective responses compared with control and fast, stimulative music. There was also a main effect of time $\left(P<0.001, \eta_{\mathrm{p}}{ }^{2}=0.34\right)$, with pairwise comparisons showing differences between time 1 (active recovery) and time 2, time 1 and time 3, time 1 and time 4 , and time 2 and time 4 of the passive recovery phase. There was no main effect of gender $\left(P=0.949, \eta_{\mathrm{p}}{ }^{2}=0.00\right)$.

Affective arousal. There was a significant main effect of condition for affective arousal $\left(P<0.001, \eta_{\mathrm{p}}{ }^{2}=0.50\right.$; see Table 1), with pairwise comparisons showing that there were differences among all three conditions. There was also a main effect of time $\left(P<0.001, \eta_{\mathrm{p}}{ }^{2}=0.66\right.$; see Table 1$)$, with pairwise comparisons indicating that there were differences between time 1 and time 2, time 1 and time 3, time 1 and time 4, time 2 and time 3, and time 2 and time 4 . There was no main effect of gender $\left(P=0.502, \eta_{\mathrm{p}}{ }^{2}=0.01\right)$.

Salivary cortisol. There was no significant main effect of condition for salivary cortisol $\left(P=0.823, \eta_{\mathrm{p}}{ }^{2}=0.01\right)$, although there was for time $\left(P=0.003, \eta_{\mathrm{p}}{ }^{2}=0.18\right.$; see Table 1$)$. Pairwise comparisons indicated a significant difference between time 1 and time 3 , time 1 and time 4 , time 2 and time 3 , and time 2 and time 4 . There was also a significant main effect of gender $\left(P=0.042, \eta_{\mathrm{p}}{ }^{2}=0.10\right)$, with pairwise comparisons indicating that the cortisol levels recorded for women were significantly lower than those for men.

HR. There was no significant main effect of condition for HR $\left(P=0.918, \eta_{\mathrm{p}}{ }^{2}=0.02\right)$ or gender $\left(P=0.259, \eta_{\mathrm{p}}{ }^{2}=\right.$ $0.03)$. There was, however, a significant main effect of time $\left(P<0.001, \eta_{\mathrm{p}}{ }^{2}=0.46\right.$; see Table 1$)$, with pairwise comparisons indicating that there were significant differences among all four time points (time 1 -time $4 ; P<0.001$ ).

sysBP. There was no significant main effect of condition for $\operatorname{sysBP}\left(P=0.064, \eta_{\mathrm{p}}{ }^{2}=0.07\right)$. There was also no main effect of time $\left(P=0.126, \eta_{\mathrm{p}}{ }^{2}=0.05\right)$ or gender $\left(P=0.419, \eta_{\mathrm{p}}{ }^{2}=0.02\right)$.

diaBP. There was no significant main effect of condition for diaBP $\left(P=0.256, \eta_{\mathrm{p}}{ }^{2}=0.03\right)$. There was also no main 
effect of time $\left(P=0.586, \eta_{\mathrm{p}}{ }^{2}=0.01\right)$ or gender $(P=0.447$, $\left.\eta_{\mathrm{p}}{ }^{2}=0.01\right)$.

\section{DISCUSSION}

The research hypothesis stating that the slow, sedative music condition would be the most efficacious in terms of expediting recovery from exhaustive exercise was only partially supported. The null hypothesis in regard to the moderating influence of gender was accepted, albeit that women seemed to show more pronounced reductions in affective arousal in the transition from active to passive recovery, and lower cortisol levels in the latter stages of passive recovery in response to the slow, sedative music condition when compared with their male counterparts. The condition-gender interaction effect for arousal was associated with a medium effect size $\left(\eta_{\mathrm{p}}{ }^{2}=0.09\right)$, whereas the time-gender interaction for cortisol was associated with a medium-to-large effect size $\left(\eta_{\mathrm{p}}{ }^{2}=0.13\right)$.

Influence of music on recovery processes. With regard to the primary research hypothesis, it would seem that the application of slow, sedative music immediately after intense exercise is beneficial from a psychological standpoint. Over time, the slow, sedative music condition elicited significantly lower affective arousal scores when compared with both the fast, stimulative and no-music control conditions $\left(\eta_{\mathrm{p}}{ }^{2}=0.22\right)$. This finding illustrates the potential salience of a musical stimulus in down-regulating arousal $(7,32)$. Overall, the slow, sedative music condition also yielded significantly higher scores for affective valence $\left(\eta_{\mathrm{p}}{ }^{2}=0.12\right)$ coupled with lower affective arousal scores $\left(\eta_{\mathrm{p}}{ }^{2}=0.50\right)$ during recovery, when compared against the other two conditions.

As suggested earlier, the two music conditions generally had a more potent influence on the arousal dimension of core affect when compared with the valence dimension $(1,19)$. Given that exercising at a severe intensity causes a sharp decline in affective valence and induces high levels of psychomotor arousal (18), environmental manipulations that assuage these effects (e.g., the present slow, sedative music condition) would be considered preferential in terms of recovery processes. Moreover, such manipulations have important implications for public health when considered in light of recent work that links exercise-related affect with adherence to exercise $(3,33,34)$.

From a physiological standpoint, the effect of our experimental manipulations on recovery is slightly less clear. Unlike previous studies $(10,35)$, which reported that slow music accelerated hemodynamic (blood pressure and HR) recovery compared with both fast music and a no-music control, the present results only show some gender differences over time $\left(\eta_{\mathrm{p}}{ }^{2}=0.12\right)$, principal among these being lower sysBP in the slow, sedative condition during the latter stages of passive recovery for men when compared with women. It is notable that the main effect of condition for sysBP was borderline nonsignificant $\left(P=0.064, \eta_{\mathrm{p}}{ }^{2}=0.07\right)$, with the trend across conditions mirroring that of past studies $(10,35)$.
The significant time-condition interaction effect for cortisol $\left(\eta_{\mathrm{p}}{ }^{2}=0.11\right)$ leads us to accept the hypothesis that slow, sedative music would elicit the most favorable psychophysiological response. In the control condition, cortisol levels remained relatively stable over time, albeit that there was a small but significant increase at time $3(+20 \mathrm{~min})$ when compared with time 1 (active recovery; see Fig. 2). The fast, stimulative condition yielded the highest cortisol level across all time points and exhibited a fairly stable profile with no significant differences across time points. Contrastingly, the slow, sedative condition yielded the lowest cortisol levels at time 1 and time 3 when compared with fast, stimulative music (see Fig. 2). Although overall, the cortisol findings are in line with the suggested delay of $\sim 20$ min required to observe a change relative to a stressor (36), it would seem that slow, sedative music serves to maintain steady cortisol levels. This contrasts with the control and fast, stimulative conditions wherein there is a rise in cortisol between time $2(+10 \mathrm{~min})$ and time $3(+20 \mathrm{~min}$; see Fig. 2). The benefits identified for the slow, sedative condition in terms of the psychological recovery profile were closely emulated in the psychophysiological data (see Figs. 2, 4).

A plausible alternative interpretation of these psychophysiological data is that heightened cortisol release immediately after exhaustive exercise is beneficial to short-term recovery; indeed, the primary physiological role of glucocorticoid secretion is to help mobilize stored energy by allocating glucose to the brain, thus increasing the chances of survival under conditions of chronic stress (37). In addition, cortisol is known to act as a potent anti-inflammatory agent (38), thus possibly negating excessive muscle inflammatory responses to exhaustive exercise. Notably, recent data have shown elevated levels of hair cortisol in endurance-trained athletes, possibly reflecting repeated activation of the hypothalamuspituitary-adrenocortical axis through physical stress of intensive training and competitive races (39). In relation to long-term training adaptations, the biological effects of cortisol on the target tissues are less clear. Intracellular bioavailability depends on tissue-specific enzymes that interconvert active cortisol to inactive cortisone, which modulates cortisol action on target cells. This process seems to be important in maintaining equilibrium during regular and intensive exercise.

The psychological findings provide support for the proposed mechanism of the brain stem reflex that is expounded in Juslin's (12) unified theory of emotional responses to music. The fundamental acoustic properties of the music played a salient role in the regulation of affective arousal (see Fig. 1). Nonetheless, this mechanism was only partially supported by the psychophysiological marker of salivary cortisol (see Fig. 2). The observed increase in cortisol levels in the fast, stimulative condition relative to slow, sedative music at time 1 (active recovery) and time 3 (+20 min) offers some support for the potential of the latter to down-regulate levels of physiological arousal. As Juslin suggests, multiple mechanisms might be activated in tandem when one listens to music; hence, there is potential to confound interpretation of psychobiological measures. 
Influence of gender. No condition-time-gender interaction was identified for any of the dependent variables. Nonetheless, a condition-gender interaction for affective arousal was evident $\left(\eta_{\mathrm{p}}{ }^{2}=0.09\right.$; see Table 1$)$. Analysis of SEs indicated that women exhibited a more pronounced reduction in arousal scores than did men in the slow, sedative music condition. Past research has shown that women have a greater tendency compared with men to use music for emotional regulation (16) and that women also benefit more from music when a complex motor task is coordinated with the rhythmical qualities of the music (40). The former, rather than the latter, might explain the moderating influence of gender that is evident among the present findings. Further research is warranted to elucidate the moderating influence of gender in the active and passive recovery that follows exhaustive exercise. In the present study, condition-gender interactions did not emerge for any physiological variables. This concurs with the findings of Savitha et al. (10), who reported no gender differences under similar circumstances. Nonetheless, the time-gender interaction effect for cortisol $\left(\eta_{\mathrm{p}}{ }^{2}=0.13\right)$ closely matches the findings of Kirschbaum et al. (41), who reported consistent gender-based differences in mean cortisol response (1.5- to twofold higher in men) in response to a psychological stressor. The time-gender interaction effect for sysBP, which showed higher levels for men at time 1 (active recovery) but higher levels for women at time 3 (+20 $\mathrm{min}$ ) and time 4 (+30 min), is slightly anomalous. This finding might suggest that men pushed harder than did women in the closing stages of the exhaustive exercise task and then recovered more effectively during the passive recovery phase.

Limitations. Although this study was designed to account for several methodological flaws evident in past research $(9,10)$, it is not without limitation. In music-related studies of this nature, it is not possible to use a double-blind approach - at the very least, the participants will know that they are being exposed to a musical stimulus - meaning that there is a possibility for experimenter effects to ensue. Moreover, participants might respond to a given musical work differently on an individual basis to how they might respond in a social context (8).

There is evidence surrounding the limitations of traditional ramp-based protocols in consistently eliciting similar $\dot{\mathrm{V}}_{2 \text { max }}$ responses, in part because the end point of the test is not known to the participant (42). Lucía et al. (43) used a similar protocol to the one used in the present study and found that only $24 \%$ of nonathlete participants elicited a $\dot{\mathrm{VO}}_{2 \max }$ "plateau" consistent with the "classical criteria" of an absolute maximal effort. We did not measure the time that it took participants to reach exhaustion and so could not subsequently use this as a possible covariate in the analyses. Inconsistent levels of fatigue and/or exhaustion may, therefore, have influenced the recovery profile of participants and created a potential source of experimental error. Nonetheless, this potential threat to internal validity was assuaged somewhat by the use of a crossover design.
Finally, the timing of trials in the present study did not fully account either for the daily diurnal variation of cortisol levels or for those associated with the female menstrual cycle (44).

Future research. To help clarify the role of recuperative music and potential moderating influence of gender on postexercise recovery, future research might focus on a number of areas in addition to the methodological limitations of the present study (i.e., blinded design, ramp test protocol, including time to exhaustion as a covariate, daily and menstrual variation in cortisol). First, the relative dearth of research on the moderating influence of gender during recovery from exhaustive exercise requires further exploration across a range of modalities that afford greater ecological validity (i.e., everyday training or competitive settings [3]). Second, other proxies of recovery, such as HR variability and electroencephalography, would help to elucidate the psychophysiological implications of recuperative music (45). Systematic attention of the underlying mechanisms that might explain the psychophysiological responses to music could facilitate the endeavors of future researchers. For example, an exploration of how specific psychoacoustic qualities of music relate to psychophysiological measures, such as salivary cortisol, might enable practitioners to target music-related interventions with greater accuracy.

\section{CONCLUSIONS}

Cortisol levels were heightened during active recovery in the fast, stimulative music condition when compared with the slow, sedative music condition, which can be interpreted as a beneficial short-term recovery process to help mobilize stored energy by allocating glucose to the brain. Ostensibly, fast, stimulative music is more beneficial than slow, sedative music in psychophysiological terms during periods of active recovery only (6). Measures of affective valence, affective arousal, and cortisol showed the slow, sedative condition to be associated with superior recovery rates during passive recovery. Slow, sedative music also seems to confer greater benefits for women than for men in regard to affective arousal during recovery. The present findings indicate that music of a slow, sedative nature can expedite the recovery processes that follow strenuous physical exercise and is particularly beneficial in terms of downregulating affective arousal.

C. I. K. received financial support from the former School of Sport and Education at Brunel University London for the cost of cortisol analyses. M. B.'s contribution was supported by a grant from the Coordination for the Improvement of Higher Education Personnel. M. H. acknowledges support from the National Institute for Health Research Leicester Biomedical Research Centre, which is a partnership between University Hospitals of Leicester NHS Trust, Loughborough University, and the University of Leicester.

The authors have no conflicts of interests to declare. The results of the present study do not constitute endorsement by the American College of Sports Medicine.

The results of the study are presented clearly, honestly, and without fabrication, falsification, or inappropriate data manipulation. 


\section{REFERENCES}

1. Jones L, Tiller NB, Karageorghis CI. Psychophysiological effects of music on acute recovery from high-intensity interval training. Physiol Behav. 2017;170:106-14.

2. Bigliassi M, León-Domínguez U, Buzzachera CF, Barreto-Silva V, Altimari LR. How does music aid $5 \mathrm{~km}$ of running? J Strength Cond Res. 2015;29(2):305-14.

3. Martin AM, Woods CB. What sustains long-term adherence to structured physical activity after a cardiac event? J Aging Phys Act. 2012;20(2):135-47.

4. Karageorghis CI, Priest D-L. Music in the exercise domain: a review and synthesis (part I). Int Rev Sport Exerc Psychol. 2012;5(1):44-66.

5. Karageorghis CI, Priest D-L. Music in the exercise domain: a review and synthesis (part II). Int Rev Sport Exerc Psychol. 2012;5(1):67-84.

6. Eliakim M, Bodner E, Eliakim A, Nemet D, Meckel Y. Effect of motivational music on lactate levels during recovery from intense exercise. J Strength Cond Res. 2012;26(1):80-6.

7. Karageorghis CI. The scientific application of music in sport and exercise: Towards a new theoretical model. In: Lane A, editor. Sport and Exercise Psychology. 2nd ed. London (UK): Routledge; 2016. pp. 277-322.

8. Karageorghis CI. Applying Music in Exercise and Sport. Champaign (IL): Human Kinetics; 2017.

9. Jing L, Xudong W. Evaluation on the effects of relaxing music on the recovery from aerobic exercise-induced fatigue. $J$ Sports Med Phys Fitness. 2008;48(1):102-6.

10. Savitha D, Mallikarjuna R, Rao C. Effect of different musical tempo on post-exercise recovery in young adults. Indian J Physiol Pharmacol. 2010;54(1):32-6.

11. Bigliassi M, Karageorghis CI, Nowicky AV, Orgs G, Wright MJ. Cerebral mechanisms underlying the effects of music during a fatiguing isometric ankle-dorsiflexion task. Psychophysiology. 2016; 53(10):1472-83.

12. Juslin PN. What does music express? Basic emotions and beyond. Front Psychol. 2013;4:e596.

13. Clark IN, Baker FA, Taylor NF. The modulating effects of music listening on health-related exercise and physical activity in adults: a systematic review and narrative synthesis. Nord J Music Ther. 2016;25(1):76-104.

14. Russell J, Barrett LF. Core affect, prototypical emotional episodes, and other things called emotion: dissecting the elephant. J Pers Soc Psychol. 1999;76(5):805-19.

15. Bishop DT, Wright MJ, Karageorghis CI. Tempo and intensity of pre-task music modulate neural activity during reactive task performance. Psychol Music. 2014;42(5):714-27.

16. North A, Hargreaves D. Music and taste. In: North A, Hargreaves D, editors. The Social and Applied Psychology of Music. Oxford (UK): Oxford University Press; 2008. pp. 75-142.

17. Thaut M. Rhythm, Music, and the Brain: Scientific Foundations and Clinical Applications. New York (NY): Routledge; 2008.

18. Ekkekakis P. Pleasure and displeasure from the body: Perspectives from exercise. Cogn Emot. 2003;17(2):213-39.

19. Ekkekakis P, Hall EE, Petruzzello SJ. The relationship between exercise intensity and affective responses demystified: To crack the 40-year-old nut, replace the 40-year-old nutcracker! Ann Behav Med. 2008;35(2):136-49.

20. Chapados C, Levitin DJ. Cross-modal interactions in the experience of musical performances: Physiological correlates. Cognition. 2008;108(3):639-51.

21. Faul F, Erdfelder E, Lang A, Buchner A. G*Power 3: a flexible statistical power analysis program for the social, behavioral, and biomedical sciences. Behav Res Methods. 2007;39(2):175-91.

22. Karageorghis CI, Terry PC. The psychophysical effects of music in sport and exercise: A review. J Sport Behav. 1997;20(1):54-68.
23. Goldberg DP, Hillier VF. A scaled version of the General Health Questionnaire. Psychol Med. 1979;9:139-45.

24. Failde I, Ramos R, Fernandez-Palacín F. Comparison between the GHQ-28 and SF-36 (MH 1-5) for the assessment of the mental health in patients with ischaemic heart disease. Eur J Epidemiol. 2000;16(4):311-6.

25. Russell JA, Weiss A, Mendelsohn GA. Affect Grid: a single-item scale of pleasure and arousal. J Pers Soc Psychol. 1989;57(3):493-502.

26. Mehrabian A, Russell J. An Approach to Environmental Psychology. Cambridge (MA): MIT Press; 1974.

27. Van Landuyt LM, Ekkekakis P, Hall EE, Petruzzello SJ. Throwing the mountains into the lakes: On the perils of nomothetic conceptioins of the exercise-affect relationship. J Sport Exerc Psychol. 2000:208-34.

28. Alessio HM, Hutchinson KM. Effects of submaximal exercise and noise exposure on hearing loss. Res Q Exerc Sport. 1991;62(4):413-9.

29. Karageorghis CI, Terry P. Inside Sport Psychology. Champaign (IL): Human Kinetics; 2011.

30. Priest D-L, Karageorghis CI. A qualitative investigation into the characteristics and effects of music accompanying exercise. Eur Phys Educ Rev. 2008;14(3):347-66.

31. Tabachnick BG, Fidell LS. Using Multivariate Statistics. 6th ed. Boston (MA): Pearson; 2014.

32. Chanda ML, Levitin DJ. The neurochemistry of music. Trends Cogn Sci. 2013;17(4):179-93.

33. Ekkekakis P, Parfitt G, Petruzzello SJ. The pleasure and displeasure people feel when they exercise at different intensities: decennial update and progress towards a tripartite rationale for exercise intensity prescription. Sports Med. 2011;41(8):641-71.

34. Lee HH, Emerson JA, Williams DM. The exercise-affectadherence pathway: An evolutionary perspective. Front Psychol. 2016;7:e1285.

35. Desai RM, Thaker RB, Patel JR, Parmar J. Effect of music on postexercise recovery rate in young healthy individuals. Int $J$ Res Med Sci. 2015;3(4):896-8.

36. Nicolson N. Handbook of Physiological Research Methods in Health Psychology. Newbury Park (CA): Sage Publications; 2008.

37. Hamer M. Psychoneuroendocrinology and physical activity. In: Acevedo E, editor. Oxford Handbook of Exercise Psychology. Oxford (UK): Oxford University Press; 2012. pp. 131-43.

38. Elenkov IJ. Glucocorticoids and the Th1/Th2 balance. Ann NY Acad Sci. 2004;1024:138-46.

39. Skoluda N, Dettenborn L, Stalder T, Kirschbaum C. Elevated hair cortisol concentrations in endurance athletes. Psychoneuroendocrinology. 2012;37(5):611-7.

40. Karageorghis CI, Priest DL, Williams LS, Hirani RM, Lannon KM, Bates BJ. Ergogenic and psychological effects of synchronous music during circuit-type exercise. Psychol Sport Exerc. 2010;11(6):551-9.

41. Kirschbaum C, Wust S, Hellhammer D. Consistent sex differences in cortisol responses to psychological stress. Psychosom Med. 1992;54(6):648-57.

42. Mauger AR, Sculthorpe N. A new $\mathrm{VO}_{2 \max }$ protocol allowing selfpacing in maximal incremental exercise. Med Sci Sport Exerc. 2010;46(1):59-63.

43. Lucía A, Rabadán M, Hoyos J, Hernández-Capilla M, Pérez M, San Juan $\mathrm{AF}$, et al. Frequency of the $\mathrm{VO}_{2 \max }$ plateau phenomenon in world-class cyclists. Int J Sports Med. 2006;27(12):984-92.

44. Wolfram M, Bellingrath S, Kudielka BM. The cortisol awakening response (CAR) across the female menstrual cycle. Psychoneuroendocrinology. 2011;36(6):905-12.

45. Bigliassi M, Karageorghis CI, Wright MJ, Orgs G, Nowicky AV. Effects of auditory stimuli on electrical activity in the brain during cycle ergometry. Physiol Behav. 2017;177:135-47. 